\title{
Constraints on Minimal SUSY models with warm dark matter neutralinos
}

\author{
Graciela Gelmini and Carlos E. Yaguna \\ Department of Physics and Astronomy, UCLA, 475 Portola Plaza, Los Angeles, CA 90095, USA
}

\begin{abstract}
If the energy density of the Universe before nucleosynthesis is dominated by a scalar field $\phi$ that decays and reheats the plasma to a low reheating temperature $T_{R H}$, neutralinos may be warm dark matter particles. We study this possibility and derive the conditions on the production mechanism and on the supersymmetric spectrum for which it is viable. Large values of the $\mu$ parameter and of the slepton masses are characteristic features of these models. We compute the expected direct detection cross sections and point out that Split-SUSY provides a natural framework for neutralino warm dark matter.
\end{abstract}

PACS numbers: 95.35.+d, 98.80.Cq. 12.60.Jv, 14.80.Ly

Thermally produced neutralinos are typical and wellmotivated cold dark matter candidates (see e.g. 1]). They are produced by scatterings in the thermal bath, then reach equilibrium and finally decouple when nonrelativistic. Non-thermally produced neutralinos, on the contrary, are usually produced as relativistic final states in the decay of heavy particles and may never reach chemical or kinetic equilibrium. As a result, they are not necessarily cold. Indeed, if they manage to keep most of their initial energy they might behave as warm dark matter suppressing the evolution of small-scale structures in the Universe.

Although consistent with the observations of the large scale structure of the Universe and the cosmic microwave background radiation anisotropies, cold dark matter models seem to have problems at galactic scales. They not only tend to form cuspy structures in the halo density profile [2] but also predict a large overabundance of small halos near galaxies such as our own [3]. Warm dark matter, with its larger free-streaming scale, may solve these problems while maintaining the celebrated success of cold dark matter models at large scales [4].

Neutralinos as warm dark matter candidates were initially discussed in Refs. [5, [6]. In this paper we will extend, in several ways, the analysis presented in those references. Ref. [5] concentrated on the cosmological aspects of this possibility, while we study a particular particle model. Reheating temperatures smaller than $5 \mathrm{MeV}$ or $2 \mathrm{MeV}$ as those found in Ref. [6] are hardly compatible with the standard cosmological scenario, which require $T_{R H}>4 \mathrm{MeV}$ [7]. We, instead take $T_{R H} \simeq 10 \mathrm{MeV}$ as a characteristic value and find the conditions on the initial energy and on the supersymmetric spectrum for which the neutralino is a warm dark matter particle. In doing so, we ignore, following Ref. [8], the naturalness argument giving up the idea that SUSY stabilizes the weak scale, notion that is the basis for Split-SUSY models. We also discuss the non-thermal production of neutralinos, as well as the implications for direct dark matter searches of neutralino warm dark matter.

We concentrate on non-standard cosmological models (see for example Ref. [9]) in which the late decay of a scalar field $\phi$ reheats the Universe to a low reheating temperature $T_{R H}$, smaller than the standard neu- tralino freeze out temperature. Such scalar fields are common in superstring models where they appear as moduli fields. These fields get mass at the low energy supersymmetry breaking scale, typically of the order of $10^{2}-10^{3} \mathrm{TeV}$. The decay of $\phi$ into radiation increases the entropy, diluting the neutralino number density. The decay of $\phi$ into supersymmetric particles, which eventually decay into neutralinos, increases the neutralino number density. We denote by $b$ the net number of neutralinos produced on average per $\phi$ decay. The number $b$ is highly model-dependent, so is the $\phi$ field mass $m_{\phi}$. They are determined by the physics of the hidden sector, by the mechanism of supersymmetry breaking, and in superstring-inspired models by the compactification mechanism [9, 10]. Thus, here we consider $b$ and $m_{\phi}$ as free parameters.

To account for the dark matter of the Universe, the neutralino relic density must be in agreement with the observed dark matter density. In low $T_{R H}$ cosmological models essentially all neutralinos can have the dark matter density provided the right combination of the following two parameters can be achieved in the high energy theory: the reheating temperature $T_{R H}$, and the ratio of the number of neutralinos produced per $\phi$ decay over the $\phi$ field mass, i.e. $b / m_{\phi}[10,11]$. We will find later the values of $T_{R H}$ and $b / m_{\phi}$ for which the neutralinos we are interested in have the right dark matter density.

A crucial quantity that distinguishes warm from cold dark matter is the free-streaming length at matterradiation equality $\lambda_{F S}$, which depends on the parameter $r_{\chi}=a(t) p_{\chi}(t) / m_{\chi}$ (see for example Refs. [5, [6] and references therein). This parameter would be the present characterictic speed of neutralinos of mass $m_{\chi}$, if their momentum $p_{\chi}$ only redshifted from neutralino production onwards $\left(a(t)\right.$ is the scale factor with $\left.a_{0}=1\right)$. During the cosmic evolution $r_{\chi}$ is constant. Structures smaller than $\lambda_{F S}$ are damped because neutralinos can freely flow out of them. N-body simulations have shown that to explain the lack of substructure in the local group, $\lambda_{F S}$ should be of order $0.1 \mathrm{Mpc}$ [4], thus $r_{\chi} \simeq 10^{-7}$.

The parameter $r_{\chi}$ also determines (for neutralinos which are relativistic at production [12]) the neutralino phase-space density $Q$. This is defined as $Q=\rho /\left\langle v^{2}\right\rangle^{3 / 2}$ where $\rho$ is the neutralino energy density and $\left\langle v^{2}\right\rangle$ is the 
mean square value of the particle velocity. In the absence of dissipation, the coarse-grained phase-space density (the quantity that can actually be observed) can only decrease from its primordial value. The observation of dwarf-spheroidal galaxies places a lower bound on $Q$ which translates into an upper bound on $r_{\chi}$ of about $2.5 \times 10^{-7}[5$. In what follows we impose the condition $r_{\chi}=10^{-7}$ to our model.

Neutralinos are produced in $\phi$ decays, thus their average initial energy is $E_{I}=m_{\phi} / N$, where $N$ is a number which depends on the production spectrum. We expect $N$ to be of order one and require $E_{I} \gg m_{\phi}$ so that neutralinos are relativistic at production. Thus, $p_{\chi} \simeq E_{I}$ at the moment of $\phi$ decay. Assuming an instantaneous $\phi$ decay at $T_{R H}$, with no subsequent entropy production, the scale factor at decay is $a=T_{0} / T_{R H}$, where $T_{0}$ is the present photon temperature, and the parameter $r_{\chi}$ in our model is $r_{\chi}=\left(T_{0} E_{I}\right) /\left(T_{R H} m_{\chi}\right)$ i.e.

$r_{\chi} \simeq 10^{-7}\left(\frac{2.3}{N}\right)\left(\frac{m_{\phi}}{10^{3} \mathrm{TeV}}\right)\left(\frac{10 \mathrm{MeV}}{T_{R H}}\right)\left(\frac{100 \mathrm{GeV}}{m_{\chi}}\right)$.

Thus, the condition $r_{\chi}=10^{-7}$ fixes $m_{\phi}$ in terms of the reheating temperature and the neutralino mass

$$
m_{\phi}=10^{3} \mathrm{TeV}\left(\frac{N}{2.3}\right)\left(\frac{m_{\chi}}{100 \mathrm{GeV}}\right)\left(\frac{T_{R H}}{10 \mathrm{MeV}}\right) .
$$

In the estimation of $r_{\chi}$ we have assumed that the neutralinos do not lose their energy in scattering processes with the thermal bath. To ensure this condition we will simply require that the interactions of neutralinos with the particles present in the plasma, $e^{ \pm}, \nu$ and $\gamma$, are out of equilibrium. Neutralino interactions are determined by the neutralino composition in terms of gauge eigenstates. The ligthest neutralino $(\chi)$ is a linear superposition of bino $\left(\tilde{B}^{0}\right)$, wino $\left(\tilde{W}^{0}\right)$ and higgsino $\left(\tilde{H}^{0}\right)$ states,

$$
\chi=N_{11} \tilde{B}^{0}+N_{12} \tilde{W}^{0}+N_{13} \tilde{H}_{d}^{0}+N_{14} \tilde{H}_{u}^{0} .
$$

According to the dominant term in Eq. (3), $\chi$ is classified as bino-like, wino-like, or higgsino-like.

Wino and higgsino-like neutralinos are always accompanied by a chargino state $\left(\chi^{ \pm}\right)$with a mass difference $\Delta m=m_{\chi^{ \pm}}-m_{\chi} \simeq \sin ^{2}\left(\theta_{W}\right)\left(M_{Z} / m_{\chi}\right)^{2} m_{\chi}$ for higgsinos and much smaller for winos. When $m_{\chi} \Delta m<E_{I} T$ relativistic neutralinos scatter inelastically (e.g. $\chi e^{-} \rightarrow$ $\chi^{-} \nu$ ) besides scattering elastically (e.g. $\chi \nu \rightarrow \chi \nu$ ) on electrons and neutrinos. Since it is not possible to simultaneously suppress the elastic and the inelastic scattering rates, wino and higgsino-like neutralinos easily lose their initial energy. In Ref. [6] was indeed pointed-out that a wino-like neutralino cannot be warm dark matter and that a higgsino-like neutralino would only be viable if $T_{R H}<2 \mathrm{MeV}$ were allowed. Bino-like neutralinos, on the other hand, couple to electrons and neutrinos mainly through slepton exchange and typically have small interaction cross sections. In general, there are no charginos close in mass to bino-like neutralinos and inelastic scatterings can be suppressed. We will therefore focus on bino-like neutralinos in the following.

In the approximation $m_{\tilde{\ell}} \gtrsim 6 T E_{I}$, which is guaranteed by the lower bound found below, the cross section for elastic bino-lepton $(\chi-\ell)$ scattering mediated by a slepton of mass $m_{\tilde{\ell}}$ is estimated to be

$$
\sigma v \simeq \frac{g^{4}}{16 \pi} \frac{E_{\ell i} E_{\ell f}}{m_{\tilde{\ell}}^{4}} .
$$

Here $g$ is the weak hypercharge coupling constant, $E_{\ell i} \simeq$ $3 T$ is the energy of the incoming thermal-bath neutrino or electron, and $E_{\ell f}$ is the energy of the outgoing lepton. We require that $\chi-\ell$ interactions are out-of-equilibrium, i.e. that the interaction rate is smaller than the expansion rate of the Universe, $\Gamma \simeq n \sigma v<H$, where $n$ is the thermal neutrino or electron number density and $H$ is the Hubble parameter. Since the interaction rate goes as $T^{5}$ and $H$ as $T^{2}$, if the interactions are out-of-equilibrium at $T_{R H}$ they will also be out of equilibrium at any later temperature $T<T_{R H}$. Replacing $E_{\ell f}$ in Eq. प by its maximum value $E_{I}=m_{\phi} / N$, we obtain that neutralino interactions are out of equilibrium at $T_{R H}$ (and subsequently) if

$$
0.1\left(\frac{m_{\phi}}{N 10^{3} \mathrm{TeV}}\right)\left(\frac{T_{R H}}{10 \mathrm{MeV}}\right)^{2}\left(\frac{40 \mathrm{TeV}}{m_{\tilde{\ell}}}\right)^{4}<1 .
$$

Sleptons therefore must be heavy.

Due to the structure of the neutralino mass matrix, bino-like neutralinos always have a small higgsino component. As a result, they also couple to fermions through $Z$ exchange. If sleptons are heavy, the $Z$-exchange diagram could give the dominant contribution to the scattering rate. In this case the neutralino cross section is

$$
\sigma v \lesssim \frac{g^{4}}{32 \pi} \tan ^{4} \theta_{W} \cos ^{2} 2 \beta \frac{E_{\ell i} E_{\ell f}}{\mu^{4}},
$$

where $\theta_{W}$ is the weak mixing angle and $\tan \beta=v_{2} / v_{1}$ is the ratio between the vacuum expectation values of the two Higgs doublets. Eq (6) holds for $m_{Z}^{2} \lesssim 6 T E_{I}$ and $m_{\chi}^{2} \lesssim 6 T E_{I}$. The latter condition, after using Eq. 2 becomes $T_{R H} / 10 \mathrm{MeV} \gtrsim 1.3\left(m_{\chi} / 100 \mathrm{GeV}\right)$. The condition $\Gamma<H$ implies for the interaction in Eq. 6 (again replacing $E_{\ell f}$ by its maximum possible value, i.e. $E_{I}$ )

$$
0.1\left(\frac{m_{\phi}}{N 10^{3} \mathrm{TeV}}\right)\left(\frac{T_{R H}}{10 \mathrm{MeV}}\right)^{2}\left(\frac{8 \mathrm{TeV}}{\mu}\right)^{4}<1 .
$$

If $m_{\chi}^{2} \gtrsim 6 T E_{I}$ (i.e. $T_{R H} / 10 M e V \lesssim 1.3\left(m_{\chi} / 100 \mathrm{GeV}\right.$ ), the right-hand side of Eq. 6 and the left-hand side of Eq. 7 must be multiplied by the factor $m_{\chi} / 3 T_{R H} E_{I}$, which yields

$$
0.1\left(\frac{m_{\chi}}{100 \mathrm{GeV}}\right)^{2}\left(\frac{T_{R H}}{10 \mathrm{MeV}}\right)\left(\frac{6 \mathrm{TeV}}{\mu}\right)^{4}<1 .
$$


In any event, a large $\mu$ parameter value is required.

Replacing Eq. 2 into Eqs. 5 and 7 and directly from Eq. 8 we obtain bounds of the slepton masses and the parameter $\mu$ which depend only on the neutralino mass and the reheating temperature,

$$
\begin{gathered}
m_{\tilde{\ell}}>18 \mathrm{TeV}\left(\frac{m_{\chi}}{100 \mathrm{GeV}}\right)^{1 / 4}\left(\frac{T_{R H}}{10 \mathrm{MeV}}\right)^{3 / 4}, \\
\mu>4 \mathrm{TeV}\left(\frac{m_{\chi}}{100 \mathrm{GeV}}\right)^{1 / 4}\left(\frac{T_{R H}}{10 \mathrm{MeV}}\right)^{3 / 4},
\end{gathered}
$$

from Eq. 7 or

$$
\mu>3 \mathrm{TeV}\left(\frac{m_{\chi}}{100 \mathrm{GeV}}\right)^{1 / 2}\left(\frac{T_{R H}}{10 \mathrm{MeV}}\right)^{1 / 4} .
$$

when Eq. 8 holds instead.

Using the cosmological lower bound $T_{R H}>4 \mathrm{MeV}$ we obtain $m_{\tilde{\ell}}>9 \mathrm{TeV}\left(m_{\chi} / 100 \mathrm{GeV}\right)^{1 / 4}$ and $\mu>2.0 \mathrm{TeV}$ $\left(m_{\chi} / 100 \mathrm{GeV}\right)^{1 / 4}$ or $\mu>2 \mathrm{TeV}\left(m_{\chi} / 100 \mathrm{GeV}\right)^{1 / 2}$.

Let us now return to the density requirement. In general there are four different ways in which the neutralino relic density $\Omega h^{2}$ depends on $T_{R H}$, in the low reheating cosmologies we are considering. The four cases have thermal or non-thermal dominant neutralino production, with or without chemical equilibrium [10, 11]. The case of interest here is that of a very low reheating temperature and high standard neutralino relic density (corresponding to small neutralino cross sections) for which the production is non-thermal without chemical equilibrium [10]. In this case neutralinos are produced in the decay of the $\phi$ field into supersymmetric particles, with subsequent fast decay of all other supersymmetric particles into neutralinos, and the production is not compensated by annihilation. This implies

$$
\frac{\Omega_{\chi} h^{2}}{0.11} \simeq 210^{3} b\left(\frac{10^{3} \mathrm{TeV}}{m_{\phi}}\right)\left(\frac{m_{\chi}}{10^{2} \mathrm{GeV}}\right)\left(\frac{T_{R H}}{10 \mathrm{MeV}}\right) .
$$

This equation fixes the value of $b / m_{\phi}$ required for neutralinos to have the dark matter density, $\Omega_{\mathrm{dm}} h^{2}=0.11^{1}$,

$$
\frac{b}{m_{\phi}} \simeq\left(\frac{510^{-7}}{\mathrm{TeV}}\right)\left(\frac{m_{\chi}}{100 \mathrm{GeV}}\right)\left(\frac{10 \mathrm{MeV}}{T_{R H}}\right)
$$

Using the program described in Ref. 11] we have followed the evolution of the relic density of some neutralinos warm dark matter as function of $T_{R H}$. This is shown in Fig. [1 We use a Minimal Supersymmetric Standard

\footnotetext{
${ }^{1} \Omega_{\mathrm{dm}} h^{2}=0.109_{-0.006}^{+0.003}$ was obtained for a $\Lambda$ CDM model with scale-invariant primordial perturbation spectrum through a global fit of cosmic microwave background, supernovae, and large scale structure data 14]
}

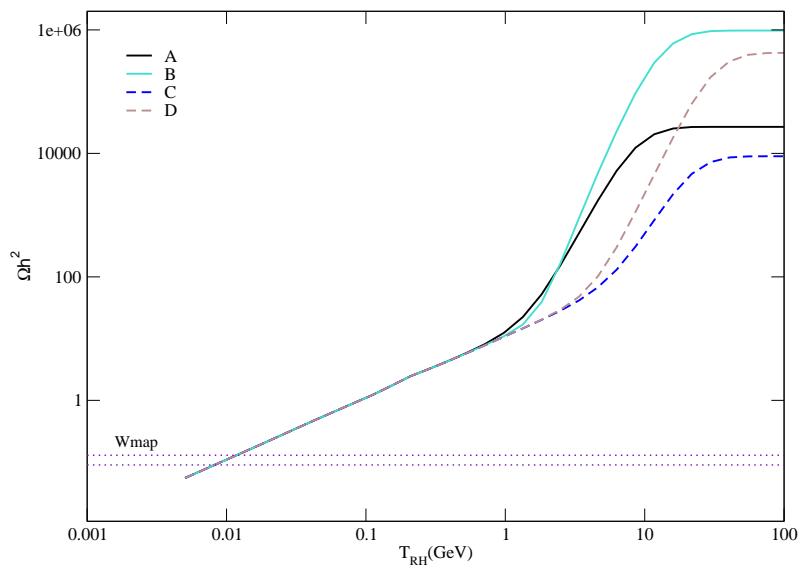

FIG. 1: The neutralino relic density as a function of $T_{R H}$ in four different models compatible with warm dark matter. In all of them $M_{2}, M_{3}, m_{A}=1 \mathrm{TeV}, \tan \beta=10, A_{t}, A_{b}=0$. In $\mathrm{A}, m_{\chi} \simeq M_{1}=100 \mathrm{GeV}, \mu=10 \mathrm{TeV}, m_{\tilde{f}}=50 \mathrm{TeV}$, and $b / m_{\phi}=4 \times 10^{-7} \mathrm{TeV}^{-1}$. In B, $m_{\chi} \simeq M_{1}=100 \mathrm{GeV}$, $\mu, m_{\tilde{f}}=100 \mathrm{TeV}$, and $b / m_{\phi}=4 \times 10^{-7} \mathrm{TeV}^{-1}$. In C, $m_{\chi} \simeq$ $M_{1}=300 \mathrm{GeV}, \mu=10 \mathrm{TeV}, m_{\tilde{f}}=50 \mathrm{TeV}$, and $b / m_{\phi}=1.3 \times$ $10^{-7} \mathrm{TeV}^{-1}$. In D, $m_{\chi} \simeq M_{1}=300 \mathrm{GeV}, \mu, m_{\tilde{f}}=100 \mathrm{TeV}$, and $b / m_{\phi}=1.3 \times 10^{-7} \mathrm{TeV}^{-1}$.

Model defined in terms of the parameter set $M_{3}, M_{2}$, $M_{1}, m_{A}, \mu, \tan \beta, m_{0}, A_{t}$, and $A_{b}$. Here $M_{i}$ are the three gaugino masses, $m_{A}$ is the mass of the pseudoscalar higgs boson, and $\tan \beta$ denotes the ratio $v_{2} / v_{1}$. The soft breaking scalar masses are defined through the simplifying ansatz $M_{Q}=M_{U}=M_{D}=M_{E}=M_{L}=m_{0}$ whereas the trilinear couplings are given by $A_{U}=\operatorname{diag}\left(0,0, \mathrm{~A}_{\mathrm{t}}\right)$, $A_{D}=\operatorname{diag}\left(0,0, \mathrm{~A}_{\mathrm{b}}\right)$, and $A_{E}=0$. All these parameters are defined at the weak scale. The models presented in Fig. 11 have $m_{\chi} \simeq M_{1}=100 \mathrm{GeV}$ (solid lines) or 300 $\mathrm{GeV}$ (dashed lines) and other parameters as mentioned in the figure caption.

Fig. 1] confirms that at low reheating temperatures the neutralino production is purely non-thermal, independently of the supersymmetric spectrum, and follows Eq. (12). Thermal effects are relevant only for $T_{R H} \gtrsim$ $1 \mathrm{GeV}$. The neutralino relic density shown in the figure correspond to $b / m_{\phi}=4 \times 10^{-7} \mathrm{TeV}^{-1}$ for $m_{\chi}=M_{1}=$ $100 \mathrm{GeV}$ (solid lines), and to $b / m_{\phi}=1.3 \times 10^{-7} \mathrm{TeV}^{-1}$ for $m_{\chi}=M_{1}=300 \mathrm{GeV}$.

Combining Eqs. 11and 12 the requirement of neutralinos to account for the whole of the dark matter and to be warm dark matter, fixes $b$ and $m_{\phi}$ (see Eq. 2) separately,

$$
\frac{\Omega_{\chi} h^{2}}{0.11} \simeq 510^{3}\left(\frac{b}{N}\right)\left(\frac{10^{-7}}{r_{\chi}}\right) .
$$

That is, fixing $\Omega_{\chi} h^{2}=0.11$ and $r_{\chi}=10^{-7}$ requires $b=$ $210^{-4} \mathrm{~N}$.

Neutralinos, therefore, can be warm dark matter particles only if slepton masses and $\mu$ are both large, above about 10 and $2 \mathrm{TeV}$ respectively. Supersymmetric mod- 


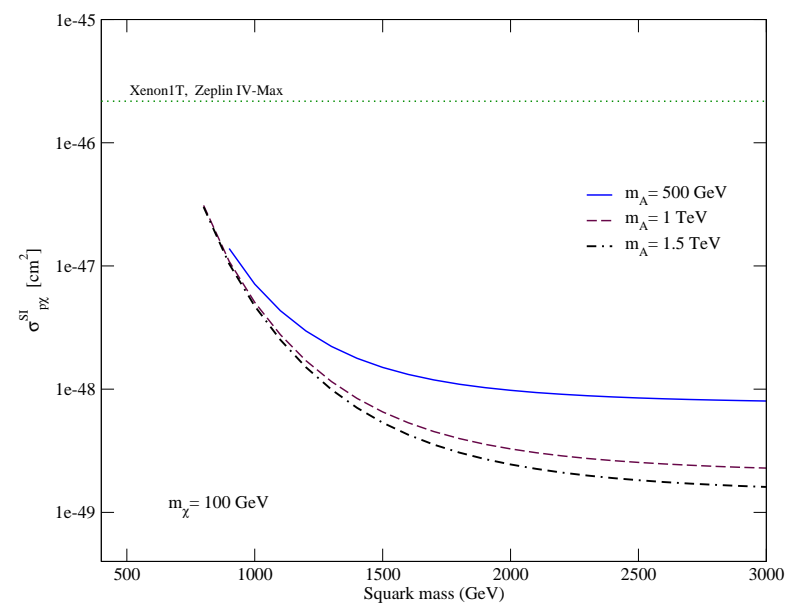

FIG. 2: Spin-independent neutralino-proton cross section for warm dark mater neutralinos as function of the squark mass, for different values of $m_{A}$, and for $M_{1}=m_{\chi}=100 \mathrm{GeV}$, $M_{2}=M_{3}=1 \mathrm{TeV}, \mu=10 \mathrm{TeV}$, slepton masses of $50 \mathrm{TeV}$ and $\tan \beta=10$. The horizontal dotted line shows the reach of Zeplin IV-MAX and Xenon 1T.

els with heavy sfermions do exist in the literature. In Split-SUSY models [8, 13, 15], all scalar superpartners are usually heavy, with a mass scale that, in principle, could go up to the GUT or the Planck scale. These models solve a number of phenomenological problems associated with ordinary supersymmetry: they alleviate proton decay, increase the mass of the Higgs boson, and minimize the flavor and CP problems. Thus, a Split-SUSY model with a bino-like LSP and $\mu \gtrsim 10 \mathrm{TeV}$ would be a perfectly viable framework for neutralino warm dark matter.

In standard cosmological models, a bino-like neutralino is allowed in Split-SUSY models only if it is almost degenerate in mass with other neutralino and chargino states $\left(M_{1} \simeq M_{2}\right.$ or $\left.M_{1} \simeq \mu\right)$ and coannihilation effects determine its relic density [16]. These constraints, however, do not hold in our model. By assuming a non-standard cosmology which allows for non-thermal production of neutralinos we have effectively enlarged the viable parameter space of Split-SUSY models.

In Split-SUSY models all scalar superpartners are usually heavy but our conditions require only that the sleptons be heavy, not necessarily the squarks. A model with light squarks would certainly be appealing from a phenomenological point of view. The most general low en- ergy spectrum of masses below a $\mathrm{TeV}$ of a model compatible with neutralino warm dark matter would consist of: the lightest neutralino, the next-to-lightest neutralino, the ligthest chargino, the three Higgs bosons, the gluino and the squarks. Such low energy spectrum is sufficiently rich to give significant signals at the LHC, particularly for light gluinos and squarks. Besides, the absence of sleptons in the low energy spectrum would be a crucial test for models with neutralino warm dark matter.

The prospects for direct detection of bino-like neutralinos depend on the neutralino-nucleon cross section, which in turn is determined by the diagrams mediated by the heavy neutral Higgs boson $(\mathrm{H})$ and squarks. We show in Fig. 2 the spin-independent neutralino-proton scattering cross section as a function of the squark mass for three different models compatible with neutralino warm dark matter, each with a different value of $m_{A}$, the pseudoscalar Higgs boson mass. The mass of heavy boson $\mathrm{H}$ is close to $m_{A}$. The lines are not continued to smaller squark masses because they enter into a region incompatible with the higgs mass bound [18] or $b \rightarrow s \gamma[19]$. The figure shows that the expected neutralino-nucleon scattering cross sections in neutralino warm dark matter models are small, between $10^{-46} \mathrm{~cm}^{2}$ and $10^{-49} \mathrm{~cm}^{2}$. Hence, the expected spin-independent neutralino-proton cross sections are below the reach of the largest dark matter detectors envisioned at present, i.e. Zeplin IV-MAX and Xenon 1 Ton [20].

In this letter we studied non-thermally produced neutralinos that are viable warm dark matter candidates, in low reheating temperature cosmological scenarios. We have shown that models compatible with neutralino warm dark matter satisfy a number of non-trivial requirements, some of them testable: the reheating temperature is low, the mass of the decaying scalar field which reheats the Universe and the number of neutralinos it produces per decay are constrained, the neutralino is binolike, slepton masses and the mu parameter are both large and the neutralino interacts too weakly to be observed in direct dark matter experiments in the near future. To validate the idea of neutralino warm dark matter, therefore, cosmological observations as well as accelerator and dark matter searches will be essential.

This work was supported in part by the US Department of Energy Grant DE-FG03-91ER40662, Task C and NASA grants NAG5-13399 and ATP03-0000-0057. We thank P. Gondolo for helpful suggestions.
[1] K. Griest and M. Kamionkowski, Phys. Rept. 333 (2000) 167.

[2] B. Moore, Nature 370, 629 (1994); R.A. Flores and J.R. Primack, Astrophys. J. 427, L1 (1994) astro-ph/9402004; J.J. Binney and N.W. Evans, Mon. Not. Roy. Astron. Soc. 327, L27 (2001) astro-ph/0108505; A.R. Zentner and J.S. Bullock, Phys.
Rev. D 66, 043003 (2002) astro-ph/0205216; J.D. Simon et al., Astrophys. J. 621, 757 (2005) astro-ph/0412035.

[3] A. A. Klypin, A. V. Kravtsov, O. Valenzuela and F. Prada, Astrophys. J. 522, 82 (1999) astro-ph/9901240; A. R. Zentner and J. S. Bullock, Astrophys. J. 598, 49 (2003) astro-ph/0304292.

[4] P. Colin, V. Avila-Reese and O. Valenzuela, Astro- 
phys. J. 542, 622 (2000) astro-ph/0004115; P. Bode, J. P. Ostriker and N. Turok, Astrophys. J. 556, 93 (2001) astro-ph/0010389.

[5] W. B. Lin, D. H. Huang, X. Zhang and R. H. Brandenberger, Phys. Rev. Lett. $86 \quad$ (2001) 954 arXiv:astro-ph/0009003.

[6] J. Hisano, K. Kohri and M. M. Nojiri, Phys. Lett. B 505, 169 (2001) arXiv:hep-ph/0011216.

[7] S. Hannestad, Phys. Rev. D 70, 043506 (2004) arXiv:astro-ph/0403291.

[8] N. Arkani-Hamed and S. Dimopoulos, JHEP 0506, 073 (2005) arXiv:hep-th/0405159.

[9] T. Moroi, M. Yamaguchi and T. Yanagida, Phys. Lett.B 342,105 (1995); M Kawasaki, T. Moroi and T. Yanagida, Phys. Lett.B 370,52 (1996); D. H. Lyth, E.D. Stewart, Phys. Rev. D 53, 1784 (1996); D. J.H. Chung, E. W. Kolb and A. Riotto, Phys. Rev. D60, 063504 (1999); T. Moroi and L. Randall, Nucl. Phys. B570, 455 (2000); G. F. Giudice, E. W. Kolb and A. Riotto, Phys. Rev. D64, 023508 (2001); R. Allahverdi and M. Drees, Phys. Rev. Lett. 89, 091302 (2002) and Phys. Rev. D66, 063513 (2002); S. Khalil, C. Muñoz and E. TorrenteLujan, New Journal of Physics 4, 27 (2002); E. TorrenteLujan, hep-ph/0210036 (2002); M. Endo, K. Hamaguchi, F. Takahashi, hep-ph/0602061 (2006); S. Nakamura, M.
Yamaguchi, hep-ph/0602081 (2006).

[10] G. B. Gelmini and P. Gondolo, arXiv:hep-ph/0602230

[11] G. Gelmini, P. Gondolo, A. Soldatenko and C. E. Yaguna, arXiv:hep-ph/0605016

[12] J. A. R. Cembranos, J. L. Feng, A. Rajaraman and F. Takayama, Phys. Rev. Lett. 95, 181301 (2005) arXiv:hep-ph/0507150.

[13] N. Arkani-Hamed, S. Dimopoulos, G. F. Giudice and A. Romanino, Nucl. Phys. B 709, 3 (2005) arXiv:hep-ph/0409232.

[14] D.N. Spergel it et al., astro-ph/0603449 (2006); http:// lambda.gsfc.nasa.gov/product/map/current/parameters.cfm.

[15] G. F. Giudice and A. Romanino, Nucl. Phys. B 699, 65 (2004) [Erratum-ibid. B 706, 65 (2005)] arXiv:hep-ph/0406088.

[16] A. Masiero, S. Profumo and P. Ullio, Nucl. Phys. B 712, 86 (2005) arXiv:hep-ph/0412058.

[17] K. Abazajian, G. M. Fuller and M. Patel, Phys. Rev. D 64, 023501 (2001) arXiv:astro-ph/0101524.

[18] [ALEPH Collaboration], arXiv:hep-ex/0602042

[19] P. Koppenburg et al. [Belle Collaboration], Phys. Rev. Lett. 93, 061803 (2004) arXiv:hep-ex/0403004.

[20] R. J. Gaitskell, Ann. Rev. Nucl. Part. Sci. 54, 315 (2004). 